\title{
Cross-Coupling Reactions of Alkenylsilanols with Fluoroalkylsulfonates
}

\author{
Scott E. Denmark* and Ramzi F. Sweis \\ Roger Adams Laboratory, Department of Chemistry, University of Illinois, Urbana, Illinois, \\ 61801
}

SUPPORTING INFORMATION

\section{General Experimental}

All reactions were performed in oven $\left(180^{\circ} \mathrm{C}\right)$ and/or flame dried glassware under an atmosphere of dry argon. All reaction temperatures corresponded to internal temperatures unless otherwise noted. All the solvents used were technical grade and distilled from the indicated drying agents: dichloromethane: $\mathrm{P}_{2} \mathrm{O}_{5}$; diethyl ether and tetrahydrofuran: $\mathrm{Na}$, benzophenone; pentane, hexane, dimethylformamide, and dimethoxyethane: $\mathrm{CaH}_{2}$; methanol: $\mathrm{Mg}(\mathrm{OMe})_{2}$; ethyl acetate: $\mathrm{K}_{2} \mathrm{CO}_{3}$. "Brine" refers to a saturated solution of $\mathrm{NaCl}$. Bulb-to-bulb distillations were performed on a Büchi GKR-50 Kugelrohr and boiling points (bp) correspond to the uncorrected, recorded air bath temperatures (ABT). All reaction temperatures correspond to internal temperatures measured with Teflon-coated thermocouples unless otherwise noted.

${ }^{1} \mathrm{H}$ and ${ }^{13} \mathrm{C}$ NMR were recorded on a Varian Unity-500 $\left(500 \mathrm{MHz}{ }^{1} \mathrm{H}, 126 \mathrm{MHz}{ }^{13} \mathrm{C}\right)$ and Varian Unity Inova $500\left(500 \mathrm{MHz}{ }^{1} \mathrm{H}, 100 \mathrm{MHz}{ }^{29} \mathrm{Si}\right)$ spectrometer in deuterochloroform unless otherwise stated using chloroform as an internal reference $\left(7.26 \mathrm{ppm},{ }^{1} \mathrm{H} ; 77.2 \mathrm{ppm},{ }^{13} \mathrm{C}\right)$. Chemical shifts are reported in ppm (d); multiplicities are indicated by s (singlet), d (doublet), q (quartet), qn (quintet), sext (sextet), m (multiplet), and br (broad). Coupling constants, $J$, are reported in Hertz $(\mathrm{Hz})$; integration is provided; and assignments are indicated.

Mass spectrometry was performed by the University of Illinois Mass Spectrometer Center. Electron impact (EI) spectra were performed on a Finnigan-MAT CH-5 spectrometer. Data are reported in the form of $\mathrm{m} / \mathrm{z}$ (intensity relative to the base peak $=100$ ). Infrared spectra (IR) were recorded on a Mattson Galaxy 5020 spectrophotometer. Peaks are reported in $\mathrm{cm}^{-1}$ with the indicated relative intensities: s (strong, 67-100\%); m (medium, 34-66\%); w (weak, 0$33 \%$ ). Elemental analyses were performed by the University of Illinois Microanalytical Service Laboratory. 
Analytical thin layer chromatography was performed on Merck Reverse-Phase C18 silica gel plates (RP C18) with F-254 indicator or Merck silica gel plates with F-254 indicator. Visualization was accomplished with UV light, an aqueous $\mathrm{KMnO}_{4}$ solution, or phosphomolybdic acid in ethanol. Column chromatography was performed using 230-400 mesh silica gel purchased from EM Science or 230-400 mesh C18 Reverse Phase (RP) silica gel purchased from Fluka.

Analytical gas chromatography (GC) was performed using a Hewlett Packard 5890 Series II Gas Chromatograph fitted with a flame ionization detector. Injections were made on a Hewlett-Packard HP-5 (50 meter) or a Hewlett-Packard Ultra-2 (50 meter) capillary column. The injector temperature was $225^{\circ} \mathrm{C}$, the detector temperature was $300{ }^{\circ} \mathrm{C}$, with a split ratio of 100:1. Retention times $\left(t_{\mathrm{R}}\right)$ and integrated ratios were obtained using Agilent Chemstation Software.

\section{Literature Preparations}

(E)-Dimethyl-(1-heptenyl)silanol, ${ }^{1}$ (Z)-dimethyl-(1-heptenyl)silanol, ${ }^{1}$ phenyl triflate, ${ }^{2}$ phenyl nonaflate, ${ }^{3}$ 1-naphthyltriflate, ${ }^{4}$ 2-methylphenyl triflate, ${ }^{5}$ 4-methoxyphenyl triflate, ${ }^{4}$ 4acetylphenyl nonaflate, ${ }^{3,6}$ and 4-t-butylcyclohex-1-enyl triflate, ${ }^{7}$ were prepared by literature methods.

\section{General Procedure I: Palladium-Catalyzed Cross-Coupling Reaction of $(E)-1$ with Phenyl Nonaflate or Phenyl Triflate.}

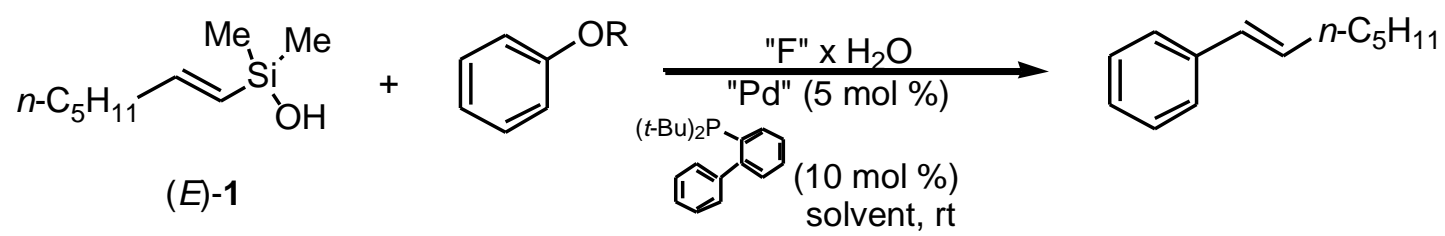

The selected tetralkylammonium fluoride ( $0.4 \mathrm{mmol}, 2.0$ equiv) was dissolved in dry dioxane or DMF $(325 \mu \mathrm{L}$ or $375 \mu \mathrm{L})$ at room temperature in a $1 \mathrm{~mL}$ conical vial with a magnetic stir bar and fitted fitted with a septum. To this solution was added the appropriate amount of water, followed by naphthalene (internal standard), di-t-butylbiphenylphosphine (BPTBP) (0.02 mmol, 0.10 equiv), the palladium bromide or palladium chloride ( $0.01 \mathrm{mmol}, 0.05$ equiv), $(E)-\mathbf{1}$ ( $0.22 \mathrm{mmol}, 1.1$ equiv), and either the phenyl nonaflate of phenyl triflate ( $2.0 \mathrm{mmol}, 1.0$ equiv). The mixture was then purged with dry argon gas via a needle though the septum. The reaction was monitored by GC analysis at certain intervals until completion. Sampling of the reaction 
was done by removing $10 \mu \mathrm{L}$ of the mixture via syringe, and adding it to a $20 \%$ solution of 2 dimethylaminoethanethiol hydrochloride to quench. The resultant mixture was then filtered through a small plug of silica gel and was eluted with $4 \mathrm{~mL}$ of diethyl ether. This sample was then analyzed by GC. Conversions were calculated relative to the internal standard. Response factors were obtained by equation 1 and are shown below:

\begin{tabular}{|c|c|c|c|c|}
\hline \multirow{2}{*}{$\begin{array}{c}\text { Response factor for } \mathrm{A}= \\
\mathrm{mg} \\
\text { naphthalene }\end{array}$} & \multicolumn{3}{|c|}{$\frac{(\text { mass } \mathrm{A} * \text { area naphthalene) }}{(\text { mass naphthalene } * \text { area } \mathrm{A})}$} & \multirow[b]{2}{*}{$\begin{array}{l}\text { response } \\
\text { factor }\end{array}$} \\
\hline & $\begin{array}{c}\text { area } \\
\text { napthalene }\end{array}$ & $\begin{array}{c}\mathrm{mg} \\
\text { product }\end{array}$ & $\begin{array}{c}\text { area } \\
\text { product }\end{array}$ & \\
\hline 75 & 2762150 & 25 & 516170 & 1.78 \\
\hline 75 & 2665150 & 25 & 517107 & 1.72 \\
\hline 75 & 2716090 & 25 & 516004 & 1.75 \\
\hline 50 & 1025480 & 50 & 512044 & 2.00 \\
\hline 50 & 1820290 & 50 & 1052880 & 1.73 \\
\hline 50 & 1674250 & 50 & 998519 & 1.68 \\
\hline 25 & 951807 & 75 & 1617150 & 1.77 \\
\hline 25 & 879020 & 75 & 1515560 & 1.74 \\
\hline 25 & 945583 & 75 & 1621300 & 1.75 \\
\hline & & & & 1.77 \\
\hline $\mathrm{mg}$ & area & $\mathrm{mg}$ & area & response \\
\hline naphthalene & napthalene & phenol & phenol & factor \\
\hline 75 & 2723480 & 75 & 2061860 & 1.32 \\
\hline 75 & 2642280 & 75 & 2003680 & 1.32 \\
\hline 75 & 2504850 & 75 & 1892990 & 1.32 \\
\hline 50 & 1802430 & 50 & 1287940 & 1.40 \\
\hline 50 & 1955660 & 50 & 1396620 & 1.40 \\
\hline 50 & 1868430 & 50 & 1334090 & 1.40 \\
\hline 25 & 967172 & 75 & 1989740 & 1.46 \\
\hline 25 & 898562 & 75 & 1847360 & 1.46 \\
\hline 25 & 1034210 & 75 & 2121570 & 1.46 \\
\hline
\end{tabular}


$t_{\mathrm{R}}$ : naphthalene, $2.98 \mathrm{~min} ; t_{\mathrm{R}}:$ phenol, $0.91 \mathrm{~min} ; t_{\mathrm{R}}:(E)$-1-heptenylbenzene, $5.92 \mathrm{~min}$ $\left(\mathrm{HP}-5,250{ }^{\circ} \mathrm{C}, 15 \mathrm{psi}\right)$

\section{Table 1, entry 1:}

Following General Procedure I, a solution of $\mathrm{TBAF} \cdot 3 \mathrm{H}_{2} \mathrm{O}$ (126 mg, $0.4 \mathrm{mmol}, 2.0$ equiv), naphthalene (15.3 mg), (E)-1 (37.9 mg, $0.22 \mathrm{mmol}, 1.1$ equiv), phenyl nonaflate $(75.2 \mathrm{mg}, 0.2$ mmol), BPTBP (6.0 mg, $0.02 \mathrm{mmol}, 0.10$ equiv), and $\mathrm{PdCl}_{2}$ (1.8 mg, $0.01 \mathrm{mmol}, 0.05$ equiv) in dioxane $(325 \mu \mathrm{L})$ was stirred at room temperature for $12 \mathrm{~h}$. An aliquot of the mixture was then taken for GC analysis.

\begin{tabular}{ccccc} 
phenol & naphthalene & product & $\%$ phenol & $\%$ product \\
\hline 64748 & 325962 & 168659 & 22.44 & 40.20
\end{tabular}

\section{Table 1, entry 2:}

Following General Procedure I, a solution of TMAF•4H $\mathrm{H}_{2} \mathrm{O}$ (66 mg, $0.4 \mathrm{mmol}, 2.0$ equiv), naphthalene (17.5 mg), (E)-1 (37.9 mg, $0.22 \mathrm{mmol}, 1.1$ equiv), phenyl nonaflate $(75.2 \mathrm{mg}, 0.2$ $\mathrm{mmol}$ ), BPTBP (6.0 mg, $0.02 \mathrm{mmol}, 0.10$ equiv), and $\mathrm{PdCl}_{2}$ (1.8 mg, $0.01 \mathrm{mmol}, 0.05$ equiv) in DMF $(325 \mu \mathrm{L})$ was stirred at room temperature for $24 \mathrm{~h}$. An aliquot of the mixture was then taken for GC analysis.

\begin{tabular}{ccccc} 
phenol & naphthalene & product & $\%$ phenol & $\%$ product \\
\hline 22646 & 554380 & 657396 & 5.28 & 105.37
\end{tabular}

\section{Table 1, entry 3:}

Following General Procedure I, a solution of $\mathrm{TBAF} \bullet 3 \mathrm{H}_{2} \mathrm{O}$ (126 mg, $0.4 \mathrm{mmol}, 2.0$ equiv), naphthalene (17.5 mg), (E)-1 (37.9 mg, $0.22 \mathrm{mmol}, 1.1$ equiv), phenyl nonaflate $(75.2 \mathrm{mg}, 0.2$ mmol), BPTBP (6.0 mg, $0.02 \mathrm{mmol}, 0.10$ equiv), and $\mathrm{PdCl}_{2}$ (1.8 mg, $0.01 \mathrm{mmol}, 0.05$ equiv) in dioxane $(325 \mu \mathrm{L})$ and water $(7.2 \mu \mathrm{L}, 0.4 \mathrm{mmol}, 2$ equiv) was stirred at room temperature for 12 h. An aliquot of the mixture was then taken for GC analysis.

\begin{tabular}{ccccc} 
phenol & naphthalene & product & $\%$ phenol & $\%$ product \\
\hline 46984 & 319442 & 254332 & 19.01 & 70.75
\end{tabular}

\section{Table 1, entry 4:}

Following General Procedure I, a solution of $\mathrm{TBAF} \cdot 3 \mathrm{H}_{2} \mathrm{O}$ (126 mg, $0.4 \mathrm{mmol}, 2.0$ equiv), naphthalene (14.7 mg), (E)-1 (37.9 mg, $0.22 \mathrm{mmol}, 1.1$ equiv), phenyl nonaflate (75.2 $\mathrm{mg}, 0.2$ 
mmol), BPTBP (6.0 mg, $0.02 \mathrm{mmol}, 0.10$ equiv), and $\mathrm{PdCl}_{2}$ (1.8 mg, $0.01 \mathrm{mmol}, 0.05$ equiv) in dioxane $(325 \mu \mathrm{L})$ and water $(21.6 \mu \mathrm{L}, 1.2 \mathrm{mmol}, 6$ equiv) was stirred at room temperature for 12 h. An aliquot of the mixture was then taken for GC analysis.

$\begin{array}{ccccc}\text { phenol } & \text { naphthalene } & \text { product } & \% \text { phenol } & \% \text { product } \\ 10880 & 298672 & 297202 & 5.17 & 97.01\end{array}$

\section{Table 1, entry 5:}

Following General Procedure I, a solution of $\mathrm{TBAF} \bullet 3 \mathrm{H}_{2} \mathrm{O}$ (126 mg, $0.4 \mathrm{mmol}, 2.0$ equiv), naphthalene (17.3 mg), (E)-1 (37.9 mg, $0.22 \mathrm{mmol}, 1.1$ equiv), phenyl nonaflate (75.2 mg, 0.2 mmol), BPTBP (6.0 mg, $0.02 \mathrm{mmol}, 0.10$ equiv), and $\mathrm{PdCl}_{2}$ (1.8 mg, $0.01 \mathrm{mmol}, 0.05$ equiv) in dioxane $(325 \mu \mathrm{L})$ and water $(36.0 \mu \mathrm{L}, 2.0 \mathrm{mmol}, 10$ equiv) was stirred at room temperature for $12 \mathrm{~h}$. An aliquot of the mixture was then taken for GC analysis.

\begin{tabular}{ccccc} 
phenol & naphthalene & product & $\%$ phenol & $\%$ product \\
\hline 4011 & 473397 & 428010 & 1.21 & 88.60
\end{tabular}

\section{Table 1, entry 6:}

Following General Procedure I, a solution of TBAF• $3 \mathrm{H}_{2} \mathrm{O}$ (126 mg, $0.4 \mathrm{mmol}, 2.0$ equiv), naphthalene (17.5 mg), (E)-1 (37.9 mg, $0.22 \mathrm{mmol}, 1.1$ equiv), phenyl nonaflate $(75.2 \mathrm{mg}, 0.2$ $\mathrm{mmol}$ ), BPTBP (6.0 mg, $0.02 \mathrm{mmol}, 0.10$ equiv), and $\mathrm{PdCl}_{2}$ (1.8 mg, $0.01 \mathrm{mmol}, 0.05$ equiv) in dioxane $(325 \mu \mathrm{L})$ and water $(50.4 \mu \mathrm{L}, 2.8 \mathrm{mmol}, 14$ equiv) was stirred at room temperature for $12 \mathrm{~h}$. An aliquot of the mixture was then taken for GC analysis.

\begin{tabular}{ccccc} 
phenol & naphthalene & product & $\%$ phenol & $\%$ product \\
\hline 1507 & 366691 & 314062 & 0.53 & 76.11
\end{tabular}

\section{Table 1, entry 7:}

Following General Procedure I, a solution of TMAF (37 mg, $0.4 \mathrm{mmol}, 2.0$ equiv), naphthalene (15.4 mg), (E)-1 (37.9 mg, $0.22 \mathrm{mmol}, 1.1$ equiv), phenyl nonaflate (75.2 $\mathrm{mg}, 0.2 \mathrm{mmol})$, BPTBP (6.0 mg, $0.02 \mathrm{mmol}, 0.10$ equiv), and $\mathrm{PdCl}_{2}(1.8 \mathrm{mg}, 0.01 \mathrm{mmol}, 0.05$ equiv) in DMF $(350 \mu \mathrm{L})$ was stirred at room temperature for $24 \mathrm{~h}$. An aliquot of the mixture was then taken for GC analysis.

\begin{tabular}{ccccc} 
phenol & naphthalene & product & $\%$ phenol & $\%$ product \\
\hline 101714 & 569770 & 10219 & 20.30 & 1.40
\end{tabular}




\section{Table 1, entry 8:}

Following General Procedure I, a solution of TMAF•4 $\mathrm{H}_{2} \mathrm{O}$ (66 mg, $0.4 \mathrm{mmol}, 2.0$ equiv), naphthalene (15.7 mg), (E)-1 (37.9 mg, $0.22 \mathrm{mmol}, 1.1$ equiv), phenyl nonaflate $(75.2 \mathrm{mg}, 0.2$ $\mathrm{mmol}$ ), BPTBP (6.0 mg, $0.02 \mathrm{mmol}, 0.10$ equiv), and $\mathrm{PdCl}_{2}$ (1.8 mg, $0.01 \mathrm{mmol}, 0.05$ equiv) in DMF $(325 \mu \mathrm{L})$ and water $(14.4 \mu \mathrm{L}, 0.8 \mathrm{mmol}, 4$ equiv) was stirred at room temperature for $24 \mathrm{~h}$. An aliquot of the mixture was then taken for GC analysis.

\begin{tabular}{rrrrr} 
phenol & naphthalene & product & $\%$ phenol & $\%$ product \\
\hline 3145 & 442023 & 561337 & 0.82 & 101.24
\end{tabular}

\section{Table 1, entry 9:}

Following General Procedure I, a solution of TMAF•4 $\mathrm{H}_{2} \mathrm{O}$ (66 mg, $0.4 \mathrm{mmol}, 2.0$ equiv), naphthalene (18.6 mg), (E)-1 (37.9 mg, $0.22 \mathrm{mmol}, 1.1$ equiv), phenyl nonaflate $(75.2 \mathrm{mg}, 0.2$ $\mathrm{mmol}$ ), BPTBP (6.0 mg, $0.02 \mathrm{mmol}, 0.10$ equiv), and $\mathrm{PdCl}_{2}$ (1.8 mg, $0.01 \mathrm{mmol}, 0.05$ equiv) in DMF $(325 \mu \mathrm{L})$ and water $(28.8 \mu \mathrm{L}, 1.6 \mathrm{mmol}, 8$ equiv) was stirred at room temperature for $24 \mathrm{~h}$. An aliquot of the mixture was then taken for GC analysis.

\begin{tabular}{ccccc} 
phenol & naphthalene & product & $\%$ phenol & $\%$ product \\
\hline 1910 & 468064 & 409018 & 0.56 & 82.53
\end{tabular}

\section{Table 1, entry 10:}

Following General Procedure I, a solution of TBAF• $3 \mathrm{H}_{2} \mathrm{O}$ (126 mg, $0.4 \mathrm{mmol}, 2.0$ equiv), naphthalene (13.7 mg), (E)-1 (37.9 mg, $0.22 \mathrm{mmol}, 1.1$ equiv), phenyl nonaflate $(75.2 \mathrm{mg}, 0.2$ mmol), BPTBP (6.0 mg, $0.02 \mathrm{mmol}, 0.10$ equiv), and PdBr $_{2}$ (2.7 mg, $0.01 \mathrm{mmol}, 0.05$ equiv) in dioxane $(325 \mu \mathrm{L})$ and water $(21.6 \mu \mathrm{L}, 1.2 \mathrm{mmol}, 6$ equiv) was stirred at room temperature for 8 h. An aliquot of the mixture was then taken for GC analysis.

\begin{tabular}{ccccc} 
phenol & naphthalene & product & $\%$ phenol & $\%$ product \\
\hline 14744 & 572692 & 795802 & 2.60 & 96.67
\end{tabular}

\section{Table 1, entry 11:}

Following General Procedure I, a solution of $\mathrm{TBAF} \cdot 3 \mathrm{H}_{2} \mathrm{O}$ (126 mg, $0.4 \mathrm{mmol}, 2.0$ equiv), naphthalene $(13.7 \mathrm{mg}),(E)-\mathbf{1}(37.9 \mathrm{mg}, 0.22 \mathrm{mmol}, 1.1 \mathrm{equiv})$, phenyl triflate (45.2 $\mathrm{mg}, 0.2$ mmol), BPTBP (6.0 mg, $0.02 \mathrm{mmol}, 0.10$ equiv), and $\operatorname{PdBr}_{2}$ (2.7 mg, $0.01 \mathrm{mmol}, 0.05$ equiv) in 
dioxane $(325 \mu \mathrm{L})$ was stirred at room temperature for $3 \mathrm{~h}$. An aliquot of the mixture was then taken for GC analysis.

\begin{tabular}{ccccc} 
phenol & naphthalene & product & $\%$ phenol & $\%$ product \\
\hline 117076 & 532928 & 597356 & 22.23 & 77.98
\end{tabular}

\section{Table 1, entry 12:}

Following General Procedure I, a solution of $\mathrm{TBAF} \bullet 3 \mathrm{H}_{2} \mathrm{O}$ (126 mg, $0.4 \mathrm{mmol}, 2.0$ equiv), naphthalene $(10.7 \mathrm{mg}),(E)-1$ (37.9 mg, $0.22 \mathrm{mmol}, 1.1$ equiv), phenyl triflate (45.2 $\mathrm{mg}, 0.2$ mmol), BPTBP (6.0 mg, $0.02 \mathrm{mmol}, 0.10$ equiv), and $\operatorname{PdBr}_{2}$ (2.7 mg, $0.01 \mathrm{mmol}, 0.05$ equiv) in dioxane $(325 \mu \mathrm{L})$ and water $(7.2 \mu \mathrm{L}, 0.4 \mathrm{mmol}, 2$ equiv) was stirred at room temperature for $8 \mathrm{~h}$. An aliquot of the mixture was then taken for GC analysis.

\begin{tabular}{ccccc} 
phenol & naphthalene & product & $\%$ phenol & $\%$ product \\
\hline 119125 & 570742 & 894570 & 16.49 & 85.16
\end{tabular}

\section{Table 1, entry 13:}

Following General Procedure I, a solution of TBAF• $3 \mathrm{H}_{2} \mathrm{O}$ (126 mg, $0.4 \mathrm{mmol}, 2.0$ equiv), naphthalene (15.7 mg), (E)-1 (37.9 mg, $0.22 \mathrm{mmol}, 1.1$ equiv), phenyl triflate (45.2 $\mathrm{mg}, 0.2$ mmol), BPTBP (6.0 mg, $0.02 \mathrm{mmol}, 0.10$ equiv), and $\operatorname{PdBr}_{2}$ (2.7 mg, $0.01 \mathrm{mmol}, 0.05$ equiv) in dioxane $(325 \mu \mathrm{L})$ and water $(21.6 \mu \mathrm{L}, 1.2 \mathrm{mmol}, 6$ equiv) was stirred at room temperature for 8 h. An aliquot of the mixture was then taken for GC analysis.

\begin{tabular}{ccccc} 
phenol & naphthalene & product & $\%$ phenol & $\%$ product \\
\hline 34217 & 531383 & 618320 & 7.47 & 92.76
\end{tabular}

\section{Table 1, entry 14:}

Following General Procedure I, a solution of $\mathrm{TBAF} \cdot 3 \mathrm{H}_{2} \mathrm{O}$ (126 mg, $0.4 \mathrm{mmol}, 2.0$ equiv), naphthalene (14.8 mg), (E)-1 (37.9 mg, $0.22 \mathrm{mmol}, 1.1$ equiv), phenyl triflate (45.2 $\mathrm{mg}, 0.2$ mmol), BPTBP (6.0 mg, $0.02 \mathrm{mmol}, 0.10$ equiv), and $\operatorname{PdBr}_{2}$ (2.7 mg, $0.01 \mathrm{mmol}, 0.05$ equiv) in dioxane $(325 \mu \mathrm{L})$ and water $(36.0 \mu \mathrm{L}, 2.0 \mathrm{mmol}, 10$ equiv) was stirred at room temperature for 8 h. An aliquot of the mixture was then taken for GC analysis.

\begin{tabular}{ccccc} 
phenol & naphthalene & product & $\%$ phenol & $\%$ product \\
\hline 11026 & 354088 & 474234 & 3.40 & 100.65
\end{tabular}


General Procedure II: Palladium-Catalyzed Cross-Coupling Reaction of $(E)$ - or (Z)Alkenylsilanols with Aryl Triflates/Nonaflates and Alkenyl Triflates.

The selected tetralkylammonium fluoride ( $4.0 \mathrm{mmol}, 2.0$ equiv) was dissolved in dry dioxane or DMF (3.25 or $3.75 \mathrm{~mL}$ ) at room temperature in a 2-necked round-bottom flask fitted to a dry argon line and fitted with a septum. To this solution was added the appropriate amount of water, followed by di-t-butylbiphenylphosphine (BPTBP) $(0.20 \mathrm{mmol}, 0.10$ equiv) and the palladium bromide $(0.10 \mathrm{mmol}, 0.05$ equiv). This mixture first was stirred at room temperature for $30 \mathrm{~min}$ when a $Z$-silanol was employed. The silanol (2.2 mmol, 1.1 equiv) and either the aryl triflate, nonaflate or alkenyl triflate $(2.0 \mathrm{mmol}, 1.0$ equiv) was then added to the mixture. When a $Z$-silanol was employed, the reaction mixture was first quenched by addition of $400 \mu \mathrm{L}$ of a $20 \%$ solution of 2-dimethylaminoethanethiol hydrochloride. It was then filtered through a short silica gel column (20 g). The plug was eluted with diethyl ether $(100 \mathrm{~mL})$ and the eluate was evaporated in vacuo. The residue was purified by column chromatography (Reverse Phase C18 or $\mathrm{SiO}_{2}, 25 \mathrm{~g}$ ) to afford the corresponding product which was further purified by bulb-to-bulb distillation.

Preparation of $(E)-1-H e p t e n y l b e n z e n e ~((E)-2 a)$ (Table 2, entry 1).

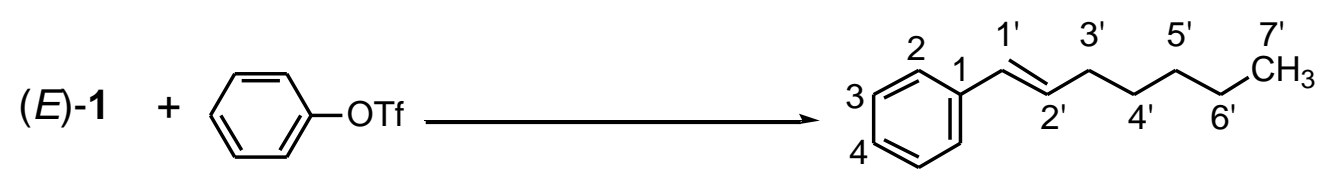

$(E)-2 a$

Following General Procedure II, a mixture of TBAF (1.26 g, $4.0 \mathrm{mmol}, 2.0$ equiv), $(E)-\mathbf{1}$ (379 mg, $2.2 \mathrm{mmol}, 1.1$ equiv), phenyl triflate (452 mg, $2.0 \mathrm{mmol})$, BPTBP (60 mg, $0.2 \mathrm{mmol}$, 0.10 equiv), and $\mathrm{PdBr}_{2}(26.6 \mathrm{mg}, 0.10 \mathrm{mmol}, 0.05$ equiv) was stirred in dioxane $(3.25 \mathrm{~mL})$ and water $(360 \mu \mathrm{L}, 20.0 \mathrm{mmol}, 10$ equiv) at room temperature for $8 \mathrm{~h}$, and then was quenched and filtered through $\mathrm{SiO}_{2}$. Purification of the residue by column chromatography (RP C18, $\left.\mathrm{MeOH} / \mathrm{H}_{2} \mathrm{O}, 9 / 1\right)$ and Kugelrohr distillation afforded $317 \mathrm{mg}(91 \%)$ of $(E)-\mathbf{2 a}$ as a colorless oil. The spectroscopic data matched those from the literature. ${ }^{1}$ 
Data for $(E)-\mathbf{2 a}$ :

bp: $\quad 160{ }^{\circ} \mathrm{C}(0.5 \mathrm{~mm} \mathrm{Hg}, \mathrm{ABT})$

${ }^{1}$ H NMR: $\quad\left(500 \mathrm{MHz}, \mathrm{CDCl}_{3}\right)$

7.35 (dt, $J=8.4,1.7,2$ H, HC(2)), 7.29 (td, $J=7.4,2.1,2$ H, HC(3)), 7.19 (tt, $J=7.3,1.3,1 \mathrm{H}, \mathrm{HC}(4)), 6.38$ (d, $\left.J=15.7,1 \mathrm{H}, \mathrm{HC}\left(1^{\prime}\right)\right), 6.24$ (dt, $J=15.8$, 6.9, $\left.1 \mathrm{H}, \mathrm{HC}\left(2^{\prime}\right)\right), 2.21$ (qd, $\left.J=7.7,1.5,2 \mathrm{H}, \mathrm{HC}\left(3^{\prime}\right)\right), 1.48$ (m, $\left.2 \mathrm{H}, \mathrm{HC}\left(4^{\prime}\right)\right)$, 1.34 ( $\mathrm{m}, 4 \mathrm{H}, \mathrm{HC}\left(5^{\prime}\right)$ and $\left.\mathrm{HC}\left(6^{\prime}\right)\right), 0.91$ (t, $\left.J=7.2,3 \mathrm{H}, \mathrm{HC}\left(7^{\prime}\right)\right)$

${ }^{13} \mathrm{C} \mathrm{NMR}: \quad\left(126 \mathrm{MHz}, \mathrm{CDCl}_{3}\right)$

$138.2,131.5,129.9,128.7,127.0,126.1,33.2,31.6,29.3,22.8,14.3$

TLC: $\quad R_{f} 0.25\left(\mathrm{MeOH} / \mathrm{H}_{2} \mathrm{O}, 9 / 1\right)[\mathrm{RP} \mathrm{C} 18, \mathrm{UV}]$

GC: $\quad t_{\mathrm{R}}(E)-\mathbf{2 a}, 8.35 \min (99.5 \%) ; t_{\mathrm{R}}(Z)-2 \mathbf{a}, 7.48 \min (0.5 \%)\left(\mathrm{U} 2,200{ }^{\circ} \mathrm{C}, 15 \mathrm{psi}\right)$

\section{Preparation of $(Z)-1-H e p t e n y l b e n z e n e ~((Z)-2 a)$ (Table 2, entry 2).}

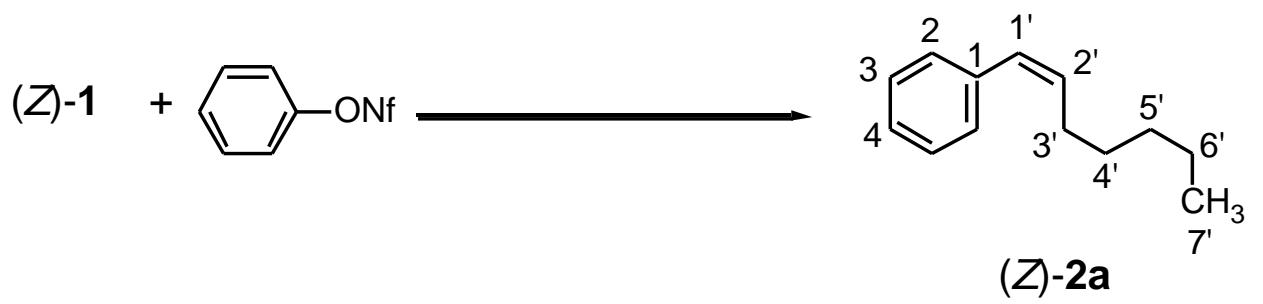

Following General Procedure II, a mixture of TBAF (1.26 g, $4.0 \mathrm{mmol}, 2.0$ equiv), $(Z)-1$ (379 mg, $2.2 \mathrm{mmol}, 1.1$ equiv), phenyl nonaflate (752 mg, $2.0 \mathrm{mmol}$ ), BPTBP (60 mg, $0.2 \mathrm{mmol}$, 0.10 equiv), and $\mathrm{PdBr}_{2}(26.6 \mathrm{mg}, 0.10 \mathrm{mmol}, 0.05$ equiv) was stirred in dioxane $(3.25 \mathrm{~mL})$ and water $(216 \mu \mathrm{L}, 12.0 \mathrm{mmol}, 6$ equiv) at room temperature for $14 \mathrm{~h}$, and then was quenched and filtered through $\mathrm{SiO}_{2}$. Purification of the residue by column chromatography (RP C18, $\mathrm{MeOH} / \mathrm{H}_{2} \mathrm{O}$, 9/1) and Kugelrohr distillation afforded $306 \mathrm{mg}(88 \%)$ of (Z)-2a as a colorless oil. The spectroscopic data matched those from the literature. ${ }^{1}$

Data for $(Z)-\mathbf{2 a}$ :

bp: $\quad 160{ }^{\circ} \mathrm{C}(0.5 \mathrm{~mm} \mathrm{Hg}, \mathrm{ABT})$

${ }^{1}$ H NMR: $\quad\left(500 \mathrm{MHz}, \mathrm{CDCl}_{3}\right)$

$7.34(\mathrm{~m}, 2 \mathrm{H}), 7.29(\mathrm{~m}, 2 \mathrm{H}), 7.22(\mathrm{tt}, J=7.2,1.5,1 \mathrm{H}), 6.41(\mathrm{~d}, J=11.8,1$ H), $5.67(\mathrm{dt}, J=11.6,7.3,1 \mathrm{H}), 2.33(\mathrm{qd}, J=7.5,1.7,2 \mathrm{H}), 1.45(\mathrm{qn}, J=7.6$, $2 \mathrm{H}), 1.32$ (m, $4 \mathrm{H}), 0.89$ (t, $J=7.1,3 \mathrm{H})$

${ }^{13} \mathrm{C} \mathrm{NMR}: \quad\left(126 \mathrm{MHz}, \mathrm{CDCl}_{3}\right)$

$138.0,133.5,129.0,128.8,128.3,126.6,31.8,29.9,28.8,22.8,14.2$ 
TLC: $\quad R_{f} 0.25\left(\mathrm{MeOH} / \mathrm{H}_{2} \mathrm{O}, 9 / 1\right)[\mathrm{RP} \mathrm{C} 18, \mathrm{UV}]$

GC: $\quad t_{\mathrm{R}}(Z)-\mathbf{2 a}, 7.43 \min (97.5 \%) ; t_{\mathrm{R}}(E)-\mathbf{2 a}, 8.24 \min (2.5 \%)\left(\mathrm{U} 2,200{ }^{\circ} \mathrm{C}, 15 \mathrm{psi}\right)$

\section{Preparation of $(E)-1-(1-H e p t e n y l) n a p h t h a l e n e ~((E)-2 b)$ (Table 2, entry 3).}
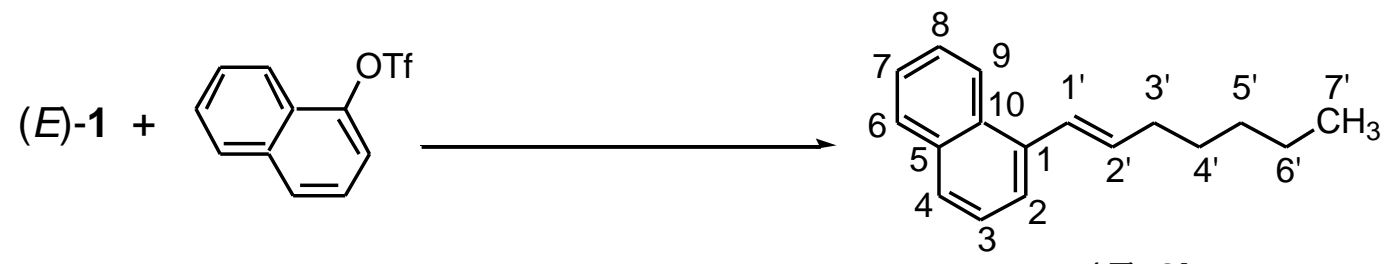

$(E)-\mathbf{2 b}$

Following General Procedure II, a mixture of TBAF (1.26 g, $4.0 \mathrm{mmol}, 2.0$ equiv), $(E)-1$ (379 mg, $2.2 \mathrm{mmol}, 1.1$ equiv), 1-naphthyl triflate (552 mg, $2.0 \mathrm{mmol}$ ), BPTBP (60 mg, 0.2 mmol, 0.10 equiv), and $\mathrm{PdBr}_{2}(26.6 \mathrm{mg}, 0.10 \mathrm{mmol}, 0.05$ equiv) was stirred in dioxane (3.25 $\mathrm{mL})$ and water $(216 \mu \mathrm{L}, 12.0 \mathrm{mmol}, 6$ equiv) at room temperature for $14 \mathrm{~h}$, and then was quenched and filtered through $\mathrm{SiO}_{2}$. Purification of the residue by column chromatography (RP $\left.\mathrm{C} 18, \mathrm{MeOH} / \mathrm{H}_{2} \mathrm{O}, 9 / 1\right)$ and Kugelrohr distillation afforded $366 \mathrm{mg}(82 \%)$ of $(E)-\mathbf{2 b}$ as a colorless oil. The spectroscopic data matched those from the literature. ${ }^{1}$

Data for $(E)-\mathbf{2 b}$ :

bp: $\quad 190{ }^{\circ} \mathrm{C}(0.5 \mathrm{~mm} \mathrm{Hg}, \mathrm{ABT})$

${ }^{1}$ H NMR: $\quad\left(500 \mathrm{MHz}, \mathrm{CDCl}_{3}\right)$

$8.13(\mathrm{~d}, J=8.2,1 \mathrm{H}), 7.84(\mathrm{dd}, J=7.7,1.9,1 \mathrm{H}), 7.74(\mathrm{~d}, J=8.1,1 \mathrm{H}), 7.56$

$(\mathrm{d}, J=7.1,1 \mathrm{H}), 7.49$ (m, $2 \mathrm{H}), 7.43$ (dd, $J=8.0,7.7,1 \mathrm{H}), 7.11(\mathrm{~d}, J=15.6$,

$1 \mathrm{H}), 6.25$ (dt, $J=15.5,7.1,1 \mathrm{H}), 2.33(\mathrm{qd}, J=7.3,1.5,2 \mathrm{H}), 1.54(\mathrm{~m}, 2 \mathrm{H})$, $1.39(\mathrm{~m}, 4 \mathrm{H}), 0.94(\mathrm{t}, J=7.2,3 \mathrm{H})$

${ }^{13} \underline{\mathrm{C} \mathrm{NMR}}: \quad\left(126 \mathrm{MHz}, \mathrm{CDCl}_{3}\right)$

136.0, 134.8, 133.8, 131.4, 128.7, 127.4, 127.1, 126.0, 125.9, 125.8, 124.2,

$123.7,33.6,31.7,29.3,22.8,14.3$

TLC: $\quad R_{f} 0.18\left(\mathrm{MeOH} / \mathrm{H}_{2} \mathrm{O}, 9 / 1\right)[\mathrm{RP} \mathrm{C} 18, \mathrm{UV}]$

GC: $\quad t_{\mathrm{R}}(E)-\mathbf{2 b}, 10.97 \min (99.1 \%) ; t_{\mathrm{R}}(Z)-\mathbf{2 b}, 9.18 \min (0.9 \%)\left(\mathrm{U} 2,250{ }^{\circ} \mathrm{C}, 15\right.$ psi) 
Preparation of (Z)-1-(1-Heptenyl)naphthalene ((Z)-2b) (Table 2, entry 4).

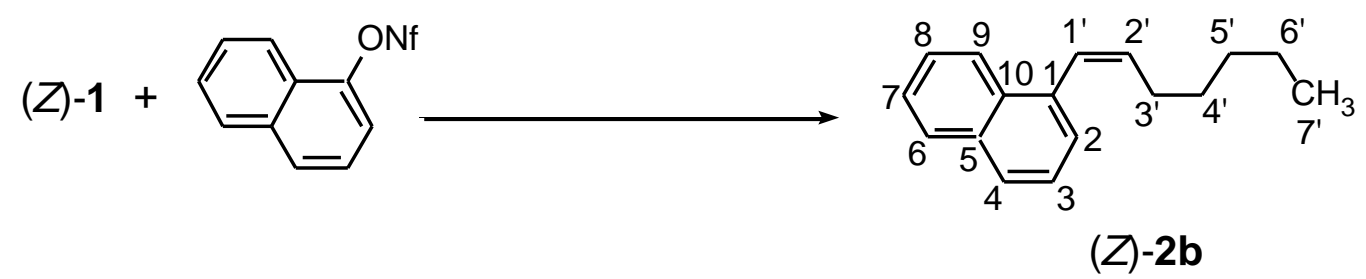

Following General Procedure II, a mixture of TBAF (1.26 g, $4.0 \mathrm{mmol}, 2.0$ equiv), (Z)-1 (379 mg, $2.2 \mathrm{mmol}, 1.1$ equiv), 1-naphthyl triflate (552 mg, $2.0 \mathrm{mmol}$ ), BPTBP (60 mg, 0.2 mmol, 0.10 equiv), and $\mathrm{PdBr}_{2}$ (26.6 mg, $0.10 \mathrm{mmol}, 0.05$ equiv) was stirred in dioxane (3.25 $\mathrm{mL})$ and water $(216 \mu \mathrm{L}, 12.0 \mathrm{mmol}, 6$ equiv) at room temperature for $15 \mathrm{~h}$, and then was quenched and filtered through $\mathrm{SiO}_{2}$. Purification of the residue by column chromatography (RP $\left.\mathrm{C} 18, \mathrm{MeOH} / \mathrm{H}_{2} \mathrm{O}, 9 / 1\right)$ and Kugelrohr distillation afforded $368 \mathrm{mg}(82 \%)$ of $(Z)-\mathbf{2 b}$ as a colorless oil. The spectroscopic data matched those from the literature. ${ }^{1}$

Data for $(Z)-\mathbf{2 a}$ :

bp: $\quad 190{ }^{\circ} \mathrm{C}(0.5 \mathrm{~mm} \mathrm{Hg}, \mathrm{ABT})$

${ }^{1}$ H NMR: $\quad\left(500 \mathrm{MHz}, \mathrm{CDCl}_{3}\right)$

$8.02(\mathrm{dd}, J=9.6,4.3,1 \mathrm{H}), 7.86(\mathrm{dd}, J=6.4,2.4,1 \mathrm{H}), 7.77(\mathrm{~d}, J=8.1,1 \mathrm{H})$, $7.50(\mathrm{~m}, 2 \mathrm{H}), 7.46(\mathrm{dd}, J=7.7,7.6,1 \mathrm{H}), 7.35(\mathrm{~d}, J=6.9,1 \mathrm{H}), 6.87(\mathrm{~d}, J=$ $11.4,1 \mathrm{H}$ ), 5.94 (dt, $J=11.5,7.5,1 \mathrm{H}), 2.15$ (qd, $J=7.3,1.5,2 \mathrm{H}$ ), 1.42 (qn, $J$ $=7.3,2 \mathrm{H}), 1.25(\mathrm{~m}, 4 \mathrm{H}), 0.83(\mathrm{t}, J=6.6,3 \mathrm{H})$

${ }^{13} \mathrm{C} \mathrm{NMR}: \quad\left(126 \mathrm{MHz}, \mathrm{CDCl}_{3}\right)$

$135.2,134.9,133.7,132.2,128.5,127.3,127.0,126.6,125.9,125.8,125.5$, $125.3,31.7,29.7,28.8,22.7,14.2$

TLC: $\quad R_{f} 0.18\left(\mathrm{MeOH} / \mathrm{H}_{2} \mathrm{O}, 9 / 1\right)[\mathrm{RP} C 18, \mathrm{UV}]$

GC: $\quad t_{\mathrm{R}}(Z)-2 \mathbf{b}, 9.29 \min (98.9 \%) ; t_{\mathrm{R}}(E)-\mathbf{2 b}, 11.10 \min (1.1 \%)\left(\mathrm{U} 2,250{ }^{\circ} \mathrm{C}, 15\right.$ psi) 
Preparation of $(E)$-1-(1-Heptenyl)-2-methylbenzene ((E)-2c) (Table 2, entry 5).
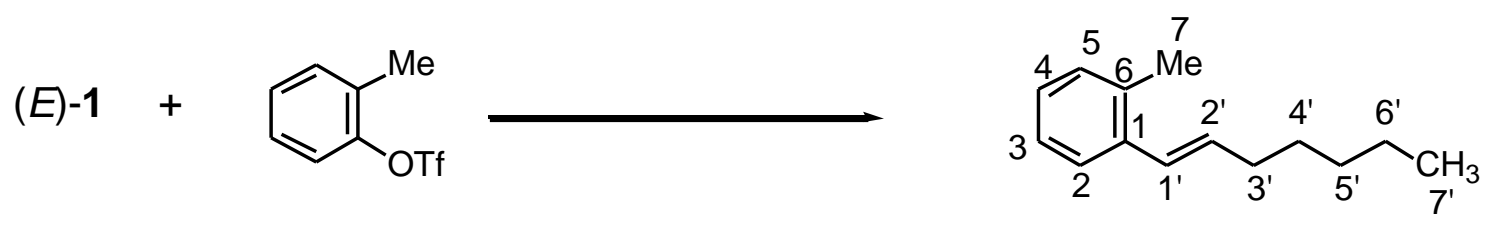

$(E)-2 c$

Following General Procedure II, a mixture of TBAF (1.26 g, $4.0 \mathrm{mmol}, 2.0$ equiv), (E)-1 (379 mg, $2.2 \mathrm{mmol}, 1.1$ equiv), 2-methylphenyl triflate (480 mg, $2.0 \mathrm{mmol}$ ), BPTBP (119.4 mg, $0.4 \mathrm{mmol}, 0.20$ equiv), and $\mathrm{PdBr}_{2}(53.2 \mathrm{mg}, 0.20 \mathrm{mmol}, 0.10$ equiv) was stirred in dioxane (3.25 $\mathrm{mL})$ and water $(216 \mu \mathrm{L}, 12.0 \mathrm{mmol}, 6.0$ equiv) at room temperature for $16 \mathrm{~h}$, and then was quenched and filtered through $\mathrm{SiO}_{2}$. Purification of the residue by column chromatography (silica gel, pentane) and Kugelrohr distillation afforded $319 \mathrm{mg}(85 \%)$ of (E)-2c as a colorless oil.

Data for $(E)-\mathbf{2 c}:$

bp: $\quad 170{ }^{\circ} \mathrm{C}(0.6 \mathrm{~mm} \mathrm{Hg}, \mathrm{ABT})$

${ }^{1}$ H NMR: $\quad\left(500 \mathrm{MHz}, \mathrm{CDCl}_{3}\right)$

$7.42(\mathrm{~d}, J=7.7,1 \mathrm{H}, \mathrm{HC}(2)), 7.13$ (m, $3 \mathrm{H}, \mathrm{HC}(3), \mathrm{HC}(4)$, and $\mathrm{HC}(5)), 6.57$ (d, $\left.J=15.7,1 \mathrm{H} \mathrm{HC}\left(1^{\prime}\right)\right), 6.10$ (dt, $\left.J=15.7,7.1,1 \mathrm{H}, \mathrm{HC}\left(2^{\prime}\right)\right), 2.34$ (s, $3 \mathrm{H}$, $\mathrm{HC}(7)$ ), 2.24 (qd, $J=7.2,1.5,2 \mathrm{H}, \mathrm{HC}\left(3^{\prime}\right)$ ), 1.50 (qn, $J=7.3,2 \mathrm{H}, \mathrm{HC}\left(4^{\prime}\right)$ ), 1.35 ( $\mathrm{m}, 4 \mathrm{H}, \mathrm{HC}\left(5^{\prime}\right)$ and $\left.\mathrm{HC}\left(6^{\prime}\right)\right), 0.92\left(\mathrm{t}, J=7.2,3 \mathrm{H}, \mathrm{HC}\left(7^{\prime}\right)\right)$

${ }^{13}$ C NMR: $\quad\left(126 \mathrm{MHz}, \mathrm{CDCl}_{3}\right)$

137.3 (C(1)), $135.1(\mathrm{C}(6)), 132.8$ (C(2')), 130.3 (C(2)), 127.7 (C(1')), 126.9 $(\mathrm{C}(3)), 126.2(\mathrm{C}(4)), 125.6(\mathrm{C}(5)), 33.5\left(\mathrm{C}\left(3^{\prime}\right)\right), 31.6\left(\mathrm{C}\left(5^{\prime}\right)\right), 29.3\left(\mathrm{C}\left(4^{\prime}\right)\right)$, $22.7\left(\mathrm{C}\left(6^{\prime}\right)\right), 20.0(\mathrm{C}(7)), 14.3\left(\mathrm{C}\left(7^{\prime}\right)\right)$

TLC: $\quad R_{f} 0.67$ (pentane) $\left[\mathrm{SiO}_{2}, \mathrm{UV}\right]$

IR: $\quad(\mathrm{NaCl})$

2956 (s), 2930 (s), 2860 (m), 1720 (s), 1603 (w), 1491 (w), 1461 (m), 1380 (w), $1288(\mathrm{w}), 1256(\mathrm{~m}), 1130(\mathrm{w}), 1078(\mathrm{~m}), 889(\mathrm{w}), 753(\mathrm{~s})$

MS: $\quad(\mathrm{EI}, 70 \mathrm{eV})$ 188 (M+, 28), 145 (5), 131 (100), 118 (52), 105 (15), 91 (22), 77 (4)

GC: $\quad t_{\mathrm{R}}(E)-\mathbf{2 c}, 76 \mathrm{~min}(99.96 \%) ; t_{\mathrm{R}}(Z)-2 \mathrm{c}, 6.95 \mathrm{~min}(0.04 \%)\left(\mathrm{U} 2,220{ }^{\circ} \mathrm{C}, 15 \mathrm{psi}\right)$ Analysis: $\quad \mathrm{C}_{14} \mathrm{H}_{20}(188.31)$
Calculated:
C, 89.30;
$\mathrm{H}, 10.70 \%$
Found:
C, 89.61;
$\mathrm{H}, 10.73 \%$ 
Preparation of (Z)-1-(1-Heptenyl)-2-methylbenzene ((Z)-2c) (Table 2, entry 6).
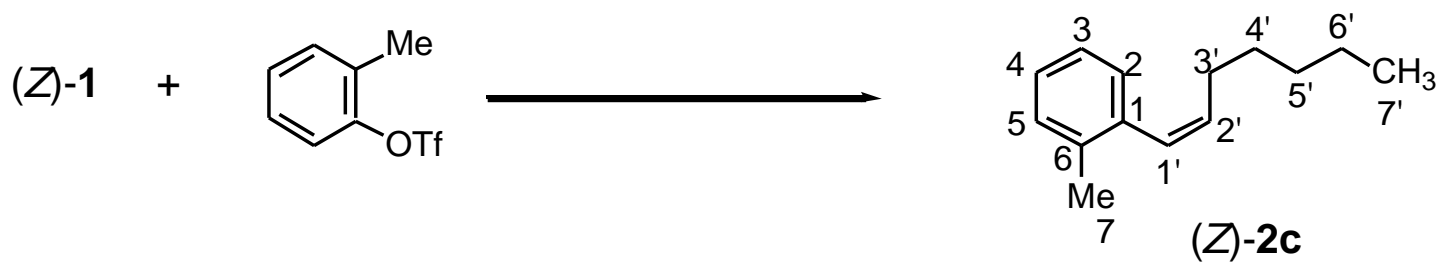

Following General Procedure II, a mixture of TBAF (1.26 g, $4.0 \mathrm{mmol}, 2.0$ equiv), (Z)-1 (379 mg, $2.2 \mathrm{mmol}, 1.1$ equiv), 2-methylphenyl triflate (480 mg, $2.0 \mathrm{mmol}$ ), BPTBP (119.4 mg, $0.4 \mathrm{mmol}, 0.20$ equiv), and $\mathrm{PdBr}_{2}(53.2 \mathrm{mg}, 0.20 \mathrm{mmol}, 0.10$ equiv) was stirred in dioxane (3.25 $\mathrm{mL})$ and water $(216 \mu \mathrm{L}, 12.0 \mathrm{mmol}, 6.0$ equiv) at room temperature for $25 \mathrm{~h}$, and then was quenched and filtered through $\mathrm{SiO}_{2}$. Purification of the residue by column chromatography (silica gel, pentane) and Kugelrohr distillation afforded $323 \mathrm{mg}(86 \%)$ of (Z)-2c as a colorless oil.

Data for $(Z)-\mathbf{2 c}$ :

bp: $\quad 170{ }^{\circ} \mathrm{C}(0.6 \mathrm{~mm} \mathrm{Hg}, \mathrm{ABT})$

${ }^{1}$ H NMR: $\quad\left(500 \mathrm{MHz}, \mathrm{CDCl}_{3}\right)$

7.16 (m, $4 \mathrm{H}, \mathrm{HC}(2), \mathrm{HC}(3), \mathrm{HC}(4)$, and $\mathrm{HC}(5)), 6.42$ (d, $J=11.6,1 \mathrm{H}$ HC(1')), 5.72 (dt, $J=11.6,7.5,1$ H, HC(2')), 2.26 (s, 3 H, HC(7)), 2.14 (qd, $J$ $\left.=7.5,1.7,2 \mathrm{H}, \mathrm{HC}\left(3^{\prime}\right)\right), 1.40\left(\mathrm{qn}, J=7.2,2 \mathrm{H}, \mathrm{HC}\left(4^{\prime}\right)\right), 1.26(\mathrm{~m}, 4 \mathrm{H}$, $\mathrm{HC}\left(5^{\prime}\right)$ and $\left.\mathrm{HC}\left(6^{\prime}\right)\right), 0.87$ (t, $\left.J=7.2,3 \mathrm{H}, \mathrm{HC}\left(7^{\prime}\right)\right)$

${ }^{13} \mathrm{C} \mathrm{NMR}: \quad\left(126 \mathrm{MHz}, \mathrm{CDCl}_{3}\right)$

137.3 (C(1)), 136.4 (C(6)), 133.2 (C(2')), 129.9 (C(2)), 129.2 (C(1')), 128.0 (C(3)), $126.9(\mathrm{C}(4)), 125.4(\mathrm{C}(5)), 31.6\left(\mathrm{C}\left(5^{\prime}\right)\right), 29.7\left(\mathrm{C}\left(4^{\prime}\right)\right), 28.5\left(\mathrm{C}\left(3^{\prime}\right)\right)$, $22.7\left(\mathrm{C}\left(6^{\prime}\right)\right), 20.1(\mathrm{C}(7)), 14.2\left(\mathrm{C}\left(7^{\prime}\right)\right)$

TLC: $\quad R_{f} 0.67$ (pentane) $\left[\mathrm{SiO}_{2}, \mathrm{UV}\right]$

IR: $\quad(\mathrm{NaCl})$

2958 (s), 2927 (s), 2857 (s), 1721 (m), 1700 (m), 1601 (w), 1486 (m), 1460

(s), 1377 (m), 1289 (w), 1254 (m), 1078 (m), 1043 (m), 785 (m), 750 (s)

MS: $\quad(\mathrm{EI}, 70 \mathrm{eV})$

188 (M+, 33), 145 (6), 131 (100), 118 (43), 105 (17), 91 (20), 77 (6)

GC: $\quad t_{\mathrm{R}}(Z)-2 \mathrm{c}, 6.94 \mathrm{~min}(99.6 \%) ; t_{\mathrm{R}}(E)-2 \mathrm{c}, 7.76 \mathrm{~min}(0.4 \%)\left(\mathrm{U} 2,220{ }^{\circ} \mathrm{C}, 15 \mathrm{psi}\right)$

Analysis: $\quad \mathrm{C}_{14} \mathrm{H}_{20}(188.31)$

Calculated: $\quad$ C, 89.30; $\mathrm{H}, 10.70 \%$

Found: $\quad$ C, $89.03 ; \quad \mathrm{H}, 10.90 \%$ 
Preparation of $(E)-1-(1-H e p t e n y l)-4-m e t h o x y b e n z e n e ~((E)-2 d)$ (Table 2, entry 7).
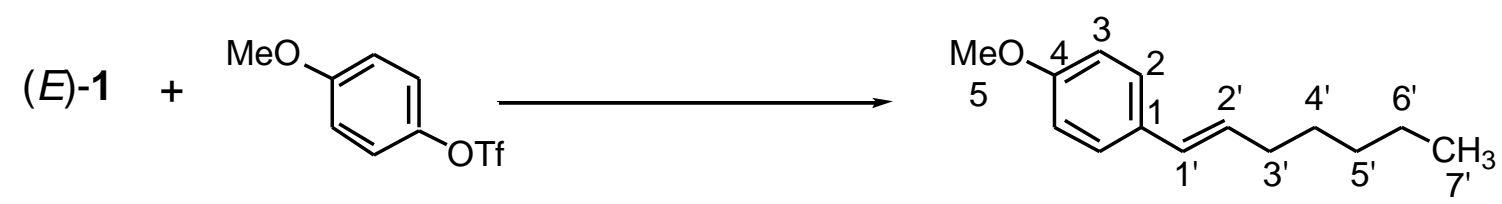

$(E)-2 d$

Following General Procedure II, a mixture of TBAF (1.26 g, $4.0 \mathrm{mmol}, 2.0$ equiv), (E)-1 (379 mg, $2.2 \mathrm{mmol}, 1.1$ equiv), 4-methoxyphenyl triflate (512 mg, $2.0 \mathrm{mmol}$ ), BPTBP (60 mg, $0.2 \mathrm{mmol}, 0.10$ equiv), and $\mathrm{PdBr}_{2}$ (26.6 mg, $0.10 \mathrm{mmol}, 0.05$ equiv) was stirred in dioxane ( 3.25 $\mathrm{mL})$ and water $(216 \mu \mathrm{L}, 12.0 \mathrm{mmol}, 6.0$ equiv) at room temperature for $5 \mathrm{~h}$, and then was quenched and filtered through $\mathrm{SiO}_{2}$. Purification of the residue by column chromatography (silica gel, pentane/ethyl acetate, 50/1) and Kugelrohr distillation afforded $382 \mathrm{mg}$ (93\%) of (E)2d as a colorless oil. The spectroscopic data matched those from the literature. ${ }^{1}$

Data for $(E)$-2d:

bp: $\quad 180{ }^{\circ} \mathrm{C}(0.5 \mathrm{~mm} \mathrm{Hg}, \mathrm{ABT})$

${ }^{1}$ H NMR: $\quad\left(500 \mathrm{MHz}, \mathrm{CDCl}_{3}\right)$

7.28 (dd, $J=6.5,2.0,2 \mathrm{H}, \mathrm{HC}(2)), 6.84(\mathrm{dd}, J=6.6,2.1,2 \mathrm{H}, \mathrm{HC}(3)), 6.32$ (d, $\left.J=15.8,1 \mathrm{H} \mathrm{HC}\left(1^{\prime}\right)\right), 6.09$ (dt, $\left.J=15.8,7.2,1 \mathrm{H}, \mathrm{HC}\left(2^{\prime}\right)\right), 3.80$ (s, $3 \mathrm{H}$, $\mathrm{HC}(5)$ ), 2.18 (qd, $J=7.4,1.3,2 \mathrm{H}, \mathrm{HC}\left(3^{\prime}\right)$ ), 1.46 (qn, $J=7.3,2 \mathrm{H}, \mathrm{HC}\left(4^{\prime}\right)$ ), 1.33 ( $\mathrm{m}, 4 \mathrm{H}, \mathrm{HC}\left(5^{\prime}\right)$ and $\left.\mathrm{HC}\left(6^{\prime}\right)\right), 0.90\left(\mathrm{t}, J=7.1,3 \mathrm{H}, \mathrm{HC}\left(7^{\prime}\right)\right)$

${ }^{13}$ C NMR: $\quad\left(126 \mathrm{MHz}, \mathrm{CDCl}_{3}\right)$

158.8 (C(4)), $131.0(\mathrm{C}(1)), 129.3\left(\mathrm{C}\left(2^{\prime}\right)\right), 129.2\left(\mathrm{C}\left(1^{\prime}\right)\right), 127.2(\mathrm{C}(2)), 114.1$

$(\mathrm{C}(3)), 55.5(\mathrm{C}(5)), 33.2\left(\mathrm{C}\left(3^{\prime}\right)\right), 31.6\left(\mathrm{C}\left(5^{\prime}\right)\right), 29.4\left(\mathrm{C}\left(4^{\prime}\right)\right), 22.8\left(\mathrm{C}\left(6^{\prime}\right)\right), 14.3$ $\left(\mathrm{C}\left(7^{\prime}\right)\right)$

TLC: $\quad R_{f} 0.31$ (pentane/ethyl acetate, $\left.50 / 1\right)\left[\mathrm{SiO}_{2}, \mathrm{UV}\right]$

GC: $\quad t_{\mathrm{R}}(E)-2 d, 10.21 \min (98.5 \%) ; t_{\mathrm{R}}(Z)-2 \mathrm{~d}, 8.99 \min (1.5 \%)\left(\mathrm{U} 2,220{ }^{\circ} \mathrm{C}, 15\right.$ psi) 
Preparation of (Z)-1-(1-Heptenyl)-4-methoxybenzene ((Z)-2d) (Table 2, entry 8).

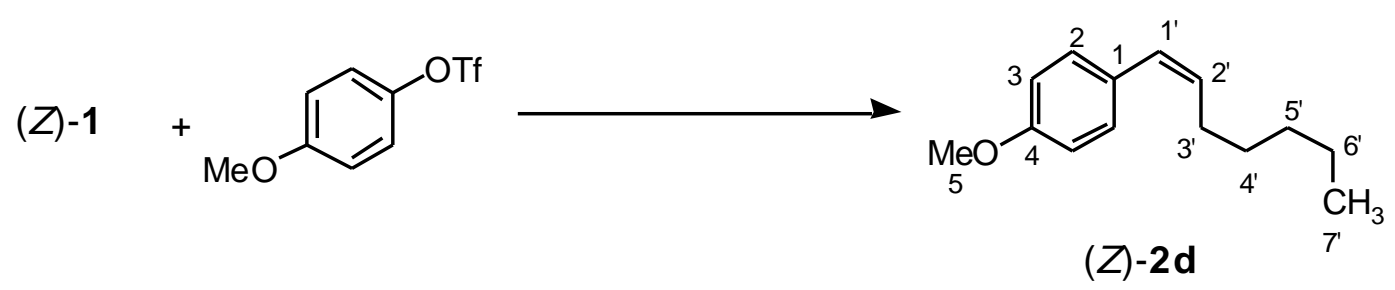

Following General Procedure II, a mixture of TBAF (1.26 g, $4.0 \mathrm{mmol}, 2.0$ equiv), (Z)-1 (379 mg, $2.2 \mathrm{mmol}, 1.1$ equiv), 4-methoxyphenyl triflate $(512 \mathrm{mg}, 2.0 \mathrm{mmol})$, BPTBP (60 mg, $0.2 \mathrm{mmol}, 0.10$ equiv), and $\mathrm{PdBr}_{2}$ (26.6 mg, $0.10 \mathrm{mmol}, 0.05$ equiv) was stirred in dioxane (3.25 $\mathrm{mL})$ and water $(216 \mu \mathrm{L}, 12.0 \mathrm{mmol}, 6.0$ equiv) at room temperature for $13 \mathrm{~h}$, and then was quenched and filtered through $\mathrm{SiO}_{2}$. Purification of the residue by column chromatography (silica gel, pentane/ethyl acetate, 50/1) and Kugelrohr distillation afforded $355 \mathrm{mg}(87 \%)$ of (Z)$\mathbf{2 d}$ as a colorless oil. The spectroscopic data matched those from the literature. ${ }^{1}$

Data for (Z)-2d:

bp: $\quad 180{ }^{\circ} \mathrm{C}(0.5 \mathrm{~mm} \mathrm{Hg}, \mathrm{ABT})$

${ }^{1}$ H NMR: $\quad\left(500 \mathrm{MHz}, \mathrm{CDCl}_{3}\right)$

$7.23(\mathrm{~d}, J=8.6,2 \mathrm{H}, \mathrm{HC}(2)), 6.87$ (d, $J=8.8,2 \mathrm{H}, \mathrm{HC}(3)), 6.34$ (d, $J=11.8$, $\left.1 \mathrm{H}, \mathrm{HC}\left(1^{\prime}\right)\right), 5.57\left(\mathrm{dt}, J=11.8,7.3,1 \mathrm{H}, \mathrm{HC}\left(2^{\prime}\right)\right), 3.82$ (s, $\left.3 \mathrm{H}, \mathrm{HC}(5)\right), 2.32$ (qd, $\left.J=7.4,1.8,2 \mathrm{H}, \mathrm{HC}\left(3^{\prime}\right)\right), 1.45$ (qn, $\left.J=7.5,2 \mathrm{H}, \mathrm{HC}\left(4^{\prime}\right)\right), 1.31(\mathrm{~m}, 4 \mathrm{H}$, $\mathrm{HC}\left(5^{\prime}\right)$ and $\left.\mathrm{HC}\left(6^{\prime}\right)\right), 0.89\left(\mathrm{t}, J=7.2,3 \mathrm{H}, \mathrm{HC}\left(7^{\prime}\right)\right)$

${ }^{13}$ C NMR: $\quad\left(126 \mathrm{MHz}, \mathrm{CDCl}_{3}\right)$

158.3 (C(4)), 131.9 (C(2')), $130.7(\mathrm{C}(1)), 130.1(\mathrm{C}(2)), 128.2$ (C(1')), 113.7 (C(3)), $55.4(\mathrm{C}(5)), 31.8\left(\mathrm{C}\left(5^{\prime}\right)\right), 29.9\left(\mathrm{C}\left(4^{\prime}\right)\right), 28.8\left(\mathrm{C}\left(3^{\prime}\right)\right), 22.8\left(\mathrm{C}\left(6^{\prime}\right)\right), 14.3$ $\left(\mathrm{C}\left(7^{\prime}\right)\right)$

TLC: $\quad R_{f} 0.31$ (pentane/ethyl acetate, $\left.50 / 1\right)\left[\mathrm{SiO}_{2}, \mathrm{UV}\right]$

GC: $\quad t_{\mathrm{R}}(Z)-2 \mathrm{~d}, 9.01 \mathrm{~min}(98.5 \%) ; t_{\mathrm{R}}(E)-\mathbf{2 d}, 10.27 \min (1.5 \%)\left(\mathrm{U} 2,220{ }^{\circ} \mathrm{C}, 15\right.$ psi) 
Preparation of $(E)$-1-[4-(1-heptenyl)phenyl]ethanone ((E)-2e) (Table 2, entry 9).

$(E)-1 \quad+$

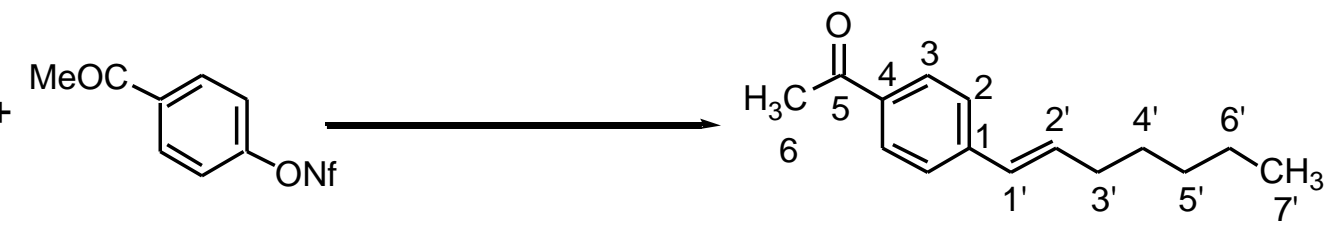

$(E)-2 e$

Following General Procedure II, a mixture of TMAF (661 mg, $4.0 \mathrm{mmol}, 2.0$ equiv), (E)1 (379 mg, $2.2 \mathrm{mmol}, 1.1$ equiv), 4-acetylphenyl nonaflate ( $837 \mathrm{mg}, 2.0 \mathrm{mmol})$, BPTBP (60 mg, $0.2 \mathrm{mmol}, 0.10$ equiv), and $\mathrm{PdBr}_{2}$ (26.6 mg, $0.10 \mathrm{mmol}, 0.05$ equiv) was stirred in DMF (2.13 $\mathrm{mL}$ ) and water ( $1.87 \mathrm{~mL}, 104 \mathrm{mmol}, 6.0$ equiv) at $50{ }^{\circ} \mathrm{C}$ for $27 \mathrm{~h}$, and then was quenched and filtered through $\mathrm{SiO}_{2}$. Purification of the residue by column chromatography (silica gel, pentane/ethyl acetate, 30/1) and Kugelrohr distillation afforded $377 \mathrm{mg}(87 \%)$ of $(E)-\mathbf{2 e}$ as a colorless oil. The spectroscopic data matched those from the literature. ${ }^{1}$

Data for $(E)-\mathbf{2 e}$ :

bp: $\quad 160{ }^{\circ} \mathrm{C}(0.5 \mathrm{~mm} \mathrm{Hg}, \mathrm{ABT})$

${ }^{1}$ H NMR: $\quad\left(500 \mathrm{MHz}, \mathrm{CDCl}_{3}\right)$

7.89 (dd, $J=8.4,2 \mathrm{H}, \mathrm{HC}(3)), 7.41$ (d, $J=8.4,2 \mathrm{H}, \mathrm{HC}(2)), 6.39$ (m, $2 \mathrm{H}$, $\mathrm{HC}\left(1^{\prime}\right)$ and $\left.\mathrm{HC}\left(2^{\prime}\right)\right), 2.58(\mathrm{~s}, 3 \mathrm{H}, \mathrm{HC}(6)), 2.24\left(\mathrm{qd}, J=7.4,1.6,2 \mathrm{H}, \mathrm{HC}\left(3^{\prime}\right)\right)$, 1.49 (qn, $\left.J=7.2,2 \mathrm{H}, \mathrm{HC}\left(4^{\prime}\right)\right), 1.33\left(\mathrm{~m}, 4 \mathrm{H}, \mathrm{HC}\left(5^{\prime}\right)\right.$ and $\left.\mathrm{HC}\left(6^{\prime}\right)\right), 0.91(\mathrm{t}, J=$ $\left.7.1,3 \mathrm{H}, \mathrm{HC}\left(7^{\prime}\right)\right)$

${ }^{13}$ C NMR: $\quad\left(126 \mathrm{MHz}, \mathrm{CDCl}_{3}\right)$

$197.9(\mathrm{C}(5)), 142.9(\mathrm{C}(4)), 135.6(\mathrm{C}(1)), 134.8\left(\mathrm{C}\left(2^{\prime}\right)\right), 129.1\left(\mathrm{C}\left(1^{\prime}\right)\right), 129.0$

$(\mathrm{C}(3)), 126.1(\mathrm{C}(2)), 33.4\left(\mathrm{C}\left(3^{\prime}\right)\right), 31.6\left(\mathrm{C}\left(5^{\prime}\right)\right), 29.0\left(\mathrm{C}\left(4^{\prime}\right)\right), 26.8(\mathrm{C}(6)), 22.7$ $\left(\mathrm{C}\left(6^{\prime}\right)\right), 14.2\left(\mathrm{C}\left(7^{\prime}\right)\right.$

TLC: $\quad R_{f} 0.25$ (pentane/ethyl acetate, $\left.50 / 1\right)\left[\mathrm{SiO}_{2}, \mathrm{UV}\right]$

GC: $\quad t_{R}(E)-2 \mathbf{e}, 9.40 \min (93.2 \%) ; t_{R}(Z)-2 \mathbf{e}, 8.91 \min (6.8 \%)\left(\mathrm{U} 2,250{ }^{\circ} \mathrm{C}, 15 \mathrm{psi}\right)$ 
Preparation of (Z)-1-[4-(1-Heptenyl)phenyl]ethanone ((Z)-2e) (Table 2, entry 10).

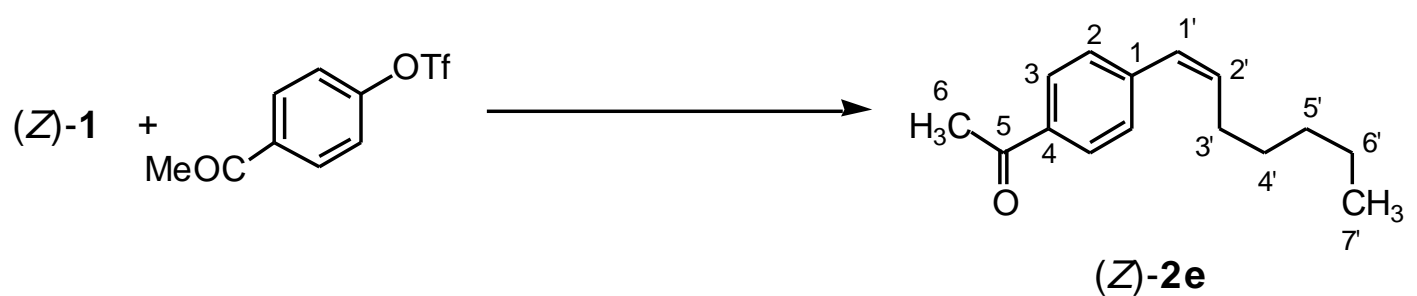

Following General Procedure II, a mixture of TMAF (661 mg, $4.0 \mathrm{mmol}, 2.0$ equiv), (E)1 (379 mg, $2.2 \mathrm{mmol}, 1.1$ equiv), 4-acetylphenyl nonaflate ( $837 \mathrm{mg}, 2.0 \mathrm{mmol}$ ), BPTBP (60 mg, $0.2 \mathrm{mmol}, 0.10$ equiv), and $\mathrm{PdBr}_{2}$ (26.6 $\mathrm{mg}, 0.10 \mathrm{mmol}, 0.05$ equiv) was stirred in DMF (2.13 $\mathrm{mL}$ ) and water ( $1.87 \mathrm{~mL}, 104 \mathrm{mmol}, 6.0$ equiv) at $50{ }^{\circ} \mathrm{C}$ for $24 \mathrm{~h}$, and then was quenched and filtered through $\mathrm{SiO}_{2}$. Purification of the residue by column chromatography (silica gel, pentane/ethyl acetate, 30/1) and Kugelrohr distillation afforded $193 \mathrm{mg}$ (91\% based on 49\% conversion in crude ${ }^{1} \mathrm{H}$ NMR) of a mixture of $(E)-2 \mathbf{e}$ and $(Z)-2 \mathbf{e}$ in an 80/20 ratio as a colorless oil. The spectroscopic data matched those from the literature. ${ }^{1}$

Data for $(E)$-2e:

See above

Data for $(Z)-2 \mathrm{e}$ :

bp: $\quad 160{ }^{\circ} \mathrm{C}(0.5 \mathrm{~mm} \mathrm{Hg}, \mathrm{ABT})$

${ }^{1}$ H NMR: $\quad\left(500 \mathrm{MHz}, \mathrm{CDCl}_{3}\right)$

7.92 (d, $J=8.3,2 \mathrm{H}, \mathrm{HC}(3)), 7.36$ (d, $J=8.5,2 \mathrm{H}, \mathrm{HC}(2)), 6.43$ (d, $J=11.7$, $\left.1 \mathrm{H}, \mathrm{HC}\left(1^{\prime}\right)\right), 5.79$ (dt, $\left.J=11.7,7.3,1 \mathrm{H}, \mathrm{HC}\left(2^{\prime}\right)\right), 2.60$ (s, $\left.3 \mathrm{H}, \mathrm{HC}(6)\right), 2.31$ (q, $\left.J=7.3,2 \mathrm{H}, \mathrm{HC}\left(3^{\prime}\right)\right), 1.46\left(\mathrm{~m}, 2 \mathrm{H}, \mathrm{HC}\left(4^{\prime}\right)\right), 1.30$ (m, $4 \mathrm{H}, \mathrm{HC}\left(5^{\prime}\right)$ and $\left.\mathrm{HC}\left(6^{\prime}\right)\right), 0.88$ (t, $\left.J=6.5,3 \mathrm{H}, \mathrm{HC}(7)\right)$

${ }^{13}$ C NMR: $\quad\left(126 \mathrm{MHz}, \mathrm{CDCl}_{3}\right)$

$197.6(\mathrm{C}(5)), 142.7(\mathrm{C}(4)), 135.7\left(\mathrm{C}\left(2^{\prime}\right)\right), 135.0(\mathrm{C}(1)), 128.8(\mathrm{C}(2)), 128.3$ $(\mathrm{C}(3)), 127.8\left(\mathrm{C}\left(1^{\prime}\right)\right), 31.5\left(\mathrm{C}\left(5^{\prime}\right)\right), 29.5\left(\mathrm{C}\left(4^{\prime}\right)\right), 28.8\left(\mathrm{C}\left(3^{\prime}\right)\right), 26.6(\mathrm{C}(6))$, $22.5\left(\mathrm{C}\left(6^{\prime}\right)\right), 14.0\left(\mathrm{C}\left(7^{\prime}\right)\right)$

TLC: $\quad R_{f} 0.25$ (pentane/ethyl acetate, $\left.50 / 1\right)\left[\mathrm{SiO}_{2}, \mathrm{UV}\right]$ 
Preparation of (E)-1-(1-Heptenyl)-4-tert-butyl-1-cyclohexene ((E)-3) (Figure \#3).

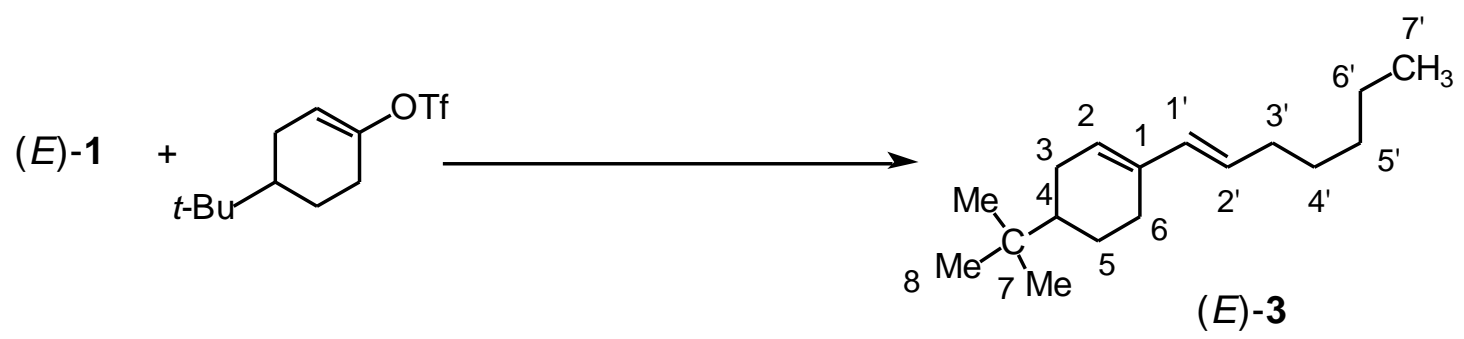

Following General Procedure II, a mixture of TBAF (1.26 g, 4.0 mmol, 2.0 equiv), (E)-1 (379 $\mathrm{mg}, 2.2 \mathrm{mmol}, 1.1$ equiv), 4-t-butylcyclohex-1-enyl triflate $(573 \mathrm{mg}, 2.0 \mathrm{mmol})$, BPTBP (60 mg, $0.2 \mathrm{mmol}, 0.10$ equiv), and $\mathrm{PdBr}_{2}$ (26.6 mg, $0.10 \mathrm{mmol}, 0.05$ equiv) was stirred in DMF (3.25 $\mathrm{mL}$ ) at $50{ }^{\circ} \mathrm{C}$ for $5 \mathrm{~h}$, and then was quenched and filtered through $\mathrm{SiO}_{2}$. Purification of the residue by gravity column chromatography ( $\mathrm{RP} \mathrm{C} 18, \mathrm{MeOH} / \mathrm{H}_{2} \mathrm{O}, 9 / 1$ ) and Kugelrohr distillation afforded $289 \mathrm{mg}(62 \%)$ of $(E)-\mathbf{3}$ as a colorless oil.

Data for $(E)-\mathbf{3}$ :

bp: $\quad 175^{\circ} \mathrm{C}(0.45 \mathrm{~mm} \mathrm{Hg}, \mathrm{ABT})$

${ }^{1}$ H NMR: $\quad\left(500 \mathrm{MHz}, \mathrm{CDCl}_{3}\right)$

$6.03\left(\mathrm{~d}, J=15.9,1 \mathrm{H}, \mathrm{HC}\left(1^{\prime}\right)\right), 5.63(\mathrm{~m}, 1 \mathrm{H}, \mathrm{HC}(2)), 5.53$ (dt, $J=15.6,6.81$ $\left.\mathrm{H}, \mathrm{HC}\left(2^{\prime}\right)\right), 1.81-2.33$ (m, 6H, $\mathrm{HC}\left(3^{\prime}\right), \mathrm{HC}(3)$ and $\left.\mathrm{HC}(6)\right), 1.10-1.42$ (m, $9 \mathrm{H}$, $\mathrm{HC}\left(4^{\prime}\right), \mathrm{HC}\left(5^{\prime}\right), \mathrm{HC}\left(6^{\prime}\right), \mathrm{HC}(4)$ and $\left.\mathrm{HC}(5)\right), 0.87$ (m, $12 \mathrm{H}, \mathrm{HC}\left(7^{\prime}\right)$ and $\mathrm{HC}(8))$

${ }^{13}$ C NMR: $\quad\left(126 \mathrm{MHz}, \mathrm{CDCl}_{3}\right)$

135.8, 133.0, 127.4, 127.3, 44.5, 36.7, 33.0, 32.4, 31.6, 29.6, 27.5, 27.4, 26.2, $22.7,14.2$

TLC: $\quad R_{f} 0.03\left(\mathrm{RP} \mathrm{C} 18, \mathrm{MeOH} / \mathrm{H}_{2} \mathrm{O}, 9 / 1\right)[\mathrm{RP} \mathrm{C} 18, \mathrm{UV}]$

IR: $\quad(\mathrm{NaCl})$

2960 (s), 2934 (s), 1721 (m), 1652 (w), 1454 (m), 1325 (m), 1289 (w), 1078 (m), $968(\mathrm{~m}), 742(\mathrm{w})$

MS: $\quad(\mathrm{EI}, 70 \mathrm{eV})$

$234(\mathrm{M}+, 38), 219$ (7), 191 (15), 177 (27), 163 (29), 149 (8), 135 (19), 121 (28), 107 (57), 93 (77), 79 (84), 67 (35), 57 (100)

GC: $\quad t_{\mathrm{R}}(E)-\mathbf{3}, 9.16 \min (98.7 \%) ; t_{\mathrm{R}}(Z)-3,8.81 \mathrm{~min}(1.3 \%)\left(\mathrm{HP}-1,15 \mathrm{psi}, 100{ }^{\circ} \mathrm{C}\right.$ (3 min), $20{ }^{\circ} \mathrm{C} / \min$ to $150{ }^{\circ} \mathrm{C}, 150{ }^{\circ} \mathrm{C}(2 \mathrm{~min}), 50{ }^{\circ} \mathrm{C} / \min$ to $\left.270{ }^{\circ} \mathrm{C}\right)$

Analysis: $\quad \mathrm{C}_{14} \mathrm{H}_{20}(234.43)$
Calculated:
C, 87.10;
$\mathrm{H}, 12.90 \%$
Found:
C, 86.93;
$\mathrm{H}, 12.94 \%$ 
Preparation of (Z)-1-(1-Heptenyl)-4-tert-butyl-1-cyclohexene ((Z)-3) (Figure \#3).

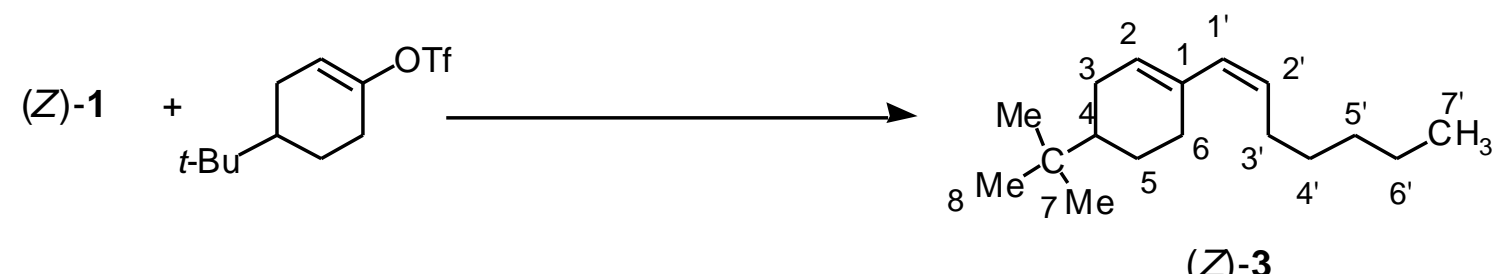

Following General Procedure II, a mixture of TBAF (1.26 g, 4.0 mmol, 2.0 equiv), (Z)-1 (379 $\mathrm{mg}, 2.2 \mathrm{mmol}, 1.1$ equiv), 4-t-butylcyclohex-1-enyl triflate $(573 \mathrm{mg}, 2.0 \mathrm{mmol})$, BPTBP (60 mg, $0.2 \mathrm{mmol}, 0.10$ equiv), and $\mathrm{PdBr}_{2}$ (26.6 mg, $0.10 \mathrm{mmol}, 0.05$ equiv) was stirred in DMF (3.25 $\mathrm{mL}$ ) at $50{ }^{\circ} \mathrm{C}$ for $12 \mathrm{~h}$, and then was quenched and filtered through $\mathrm{SiO}_{2}$. Purification of the residue by gravity column chromatography (RP C18, $\mathrm{MeOH} / \mathrm{H}_{2} \mathrm{O}, 9 / 1$ ) and Kugelrohr distillation afforded $303 \mathrm{mg}(65 \%)$ of $(Z)-\mathbf{3}$ as a colorless oil. Purity was assayed at $>97 \%$ by GC (small disiloxane contaminant) due to difficulty obtaining a sample with suitable microanalytical standards.

Data for $(Z)-3$ :

bp: $\quad 175^{\circ} \mathrm{C}(0.45 \mathrm{~mm} \mathrm{Hg}, \mathrm{ABT})$

${ }^{1}$ H NMR: $\quad\left(500 \mathrm{MHz}, \mathrm{CDCl}_{3}\right)$

$5.75\left(\mathrm{~d}, J=11.8,1 \mathrm{H}, \mathrm{HC}\left(1^{\prime}\right)\right), 5.63(\mathrm{~m}, 1 \mathrm{H}, \mathrm{HC}(2)), 5.26$ (dt, $J=11.8,7.51$ H, HC(2')), 1.81-2.32 (m, 6 H, HC(3'), HC(3) and $\mathrm{HC}(6)), 1.10-1.42$ (m, 9 H, $\mathrm{HC}\left(4^{\prime}\right), \mathrm{HC}\left(5^{\prime}\right), \mathrm{HC}\left(6^{\prime}\right), \mathrm{HC}(4)$ and $\left.\mathrm{HC}(5)\right), 0.87\left(\mathrm{~m}, 12 \mathrm{H}, \mathrm{HC}\left(7^{\prime}\right)\right.$ and $\mathrm{HC}(8))$

${ }^{13}$ C NMR: $\quad\left(126 \mathrm{MHz}, \mathrm{CDCl}_{3}\right)$

135.7, 131.3, 130.0, 127.8, 43.9, 32.4, 31.8, 30.7, 30.3, 29.9, 29.1, 27.4, 27.3, $24.5,22.8,14.2$

TLC: $\quad R_{f} 0.03\left(\mathrm{RP} \mathrm{C} 18, \mathrm{MeOH} / \mathrm{H}_{2} \mathrm{O}, 9 / 1\right)[\mathrm{RP} \mathrm{C} 18, \mathrm{UV}]$

IR: $\quad(\mathrm{NaCl})$

2957 (s), 2946 (s), 1708 (m), 1631 (w), 1463 (m), 1324 (m), 1292 (w), 1078 (m), $952(\mathrm{~m}), 720(\mathrm{w})$

MS: $\quad(\mathrm{EI}, 70 \mathrm{eV})$

234 (M+, 36), 219 (7), 191 (17), 177 (25), 163 (27), 149 (8), 135 (18), 121 (27), 107 (49), 93 (64), 79 (71), 67 (35), 57 (100) 
HRMS: $\quad(\mathrm{EI})$

Calcd. For $\left(\mathrm{C}_{17} \mathrm{H}_{30}\right): \quad 234.2348$

Found: $\quad 234.2354$

GC: $\quad t_{\mathrm{R}}(Z)-3,8.86 \min (96.6 \%) ; t_{\mathrm{R}}(E)-3,9.21 \min (3.4 \%)\left(\mathrm{HP}-1,15 \mathrm{psi}, 100{ }^{\circ} \mathrm{C}\right.$ (3 min), $20{ }^{\circ} \mathrm{C} / \min$ to $150{ }^{\circ} \mathrm{C}, 150{ }^{\circ} \mathrm{C}(2 \mathrm{~min}), 50{ }^{\circ} \mathrm{C} / \mathrm{min}$ to $270{ }^{\circ} \mathrm{C}$. $)$

\section{References}

(1) Denmark, S. E.; Wehrli, D. Org. Lett. 2000, 2, 565.

(2) (a) Anders, E.; Stanowiak, M. Synthesis 1984, 1039. (b) Mowery, M. E.; DeShong, P. J. Org. Chem. 1999, 64, 3266.

(3) Rottlander, M.; Knochel, P. J. Org. Chem. 1998, 63, 203.

(4) Echavarren, A. M.; Stille, J. K. J. Am. Chem. Soc. 1987, 109, 5478.

(5) Cabri, W.; Candiani, I.; Bedeschi, A.; Penco, S. J. Org. Chem. 1992, 57, 1481.

(6) Nishihara, Y.; Ikegashira, K.; Hirabayashi, K.; Ando, J.; Mori, A.; Hiyama, T. J. Org. Chem. 2000, 65, 1780.

(7) Scott, W. J.; Stille, J. K. J. Am. Chem Soc. 1986, 108, 3033. 